\title{
Lecture littéraire et imagination éthique
}

Literary Reading and Ethical Imagination

\section{Marion Sauvaire}

\section{OpenEdition}

Journals

Édition électronique

URL : http://journals.openedition.org/recherchestravaux/1598

DOI : 10.4000/recherchestravaux.1598

ISSN : 1969-6434

\section{Éditeur}

UGA Éditions/Université Grenoble Alpes

\section{Édition imprimée}

ISBN : 978-2-37747-098-3

ISSN : 0151-1874

\section{Référence électronique}

Marion Sauvaire, «Lecture littéraire et imagination éthique », Recherches \& Travaux [En ligne], 94 | 2019, mis en ligne le 20 juin 2019, consulté le 08 septembre 2020. URL : http://journals.openedition.org/ recherchestravaux/1598; DOI : https://doi.org/10.4000/recherchestravaux.1598

Ce document a été généré automatiquement le 8 septembre 2020

(C) Recherches \& Travaux 


\title{
Lecture littéraire et imagination éthique
}

\author{
Literary Reading and Ethical Imagination
}

Marion Sauvaire

Le défaut de réponse aux dilemmes d'une époque

est peut-être l'arme la plus efficace dont dispose

la littérature

pour agir sur les mœurs et changer la pratique.

(P. Ricœur, Temps et récit III ${ }^{1}$ )

Cette contribution présente et problématise certains fondements théoriques de la discussion actuelle, dans le domaine de la didactique de la littérature, sur les enjeux éthiques de l'enseignement de la lecture littéraire. Cette réflexion collective se nourrit, entre autres influences, de la réception renouvelée d'essais pour la plupart anglophones et déjà anciens, émanant de la critique littéraire et de la théorie de la lecture, réunis sous l'étiquette $d u$ "tournant éthique». Actualiser cette tradition critique dans un contexte culturel et social où la question des valeurs est particulièrement clivée et dans un domaine scientifique en éducation ne va pas sans soulever de riches controverses. Trois ensembles de questions peuvent être identifiés ${ }^{2}$ : enseigner l'éthique dans la littérature ou comment transposer une poétique des valeurs dans l'enseignement de la littérature ? Enseigner l'éthique par la littérature, autrement dit, comment utiliser la littérature pour transmettre une conception de la vie bonne, une morale particulière (celle de l'école républicaine) et des procédures délibératives de résolutions de problèmes éthiques par la discussion littéraire? Enseigner une éthique de la lecture littéraire, c'est-à-dire, principalement dans la foulée des approches subjectives, comment favoriser l'investissement des élèves comme sujets éthiques dans la lecture?

2 Chacune de ces questions semble soulever son lot de réserves. Le rejet d'une conception formaliste de la lecture comme jeu poétique sur les valeurs textuelles qui n'engagerait pas le lecteur comme sujet éthique, la critique d'un retour possible de 
l'instrumentalisation moralisante, voire moralisatrice, de l'enseignement de la littérature, la mise en garde à l'endroit d'une valorisation de la diversité interprétative qui verserait dans un relativisme bienpensant («tout se vaut») sont autant de préoccupations exprimées dans notre communauté de chercheurs à propos des relations entre enseignement de la littérature et visée éthique. Nous proposons de les examiner sous un angle épistémologique, en nous concentrant sur les conceptions sous-jacentes du pluralisme moral qui les irriguent. Nous pensons en effet qu'elles convoquent, souvent implicitement, des conceptions différentes de ce que devrait être une éducation au pluralisme moral dans et par l'enseignement de la lecture littéraire ${ }^{3}$.

Dans une première partie, nous reprenons succinctement les distinctions établies par le philosophe de l'éducation, Georges Leroux, concernant les notions de diversité, de pluralisme et de relativisme moral ${ }^{4}$. Cette clarification faite, nous posons la question qui constitue l'horizon de cet article : comment penser l'enseignement de la lecture littéraire, dans une visée d'éducation au pluralisme moral, qui soit dégagée de tout moralisme a priori?

Notre contribution à l'élucidation de cette question complexe se limitera très modestement à une relecture de Soi-même comme un autre de Paul Ricœur ${ }^{5}$. Dans cet ouvrage, Ricœur a consacré à sa théorie de la lecture une place charnière entre la constitution de soi (identité narrative) et la visée éthique. La notion d'identité narrative est une référence partagée par plusieurs didacticiens de la littérature pour construire celle de sujet lecteur. Étant bien connue, elle sera présentée très succinctement. Par contre, le volet éthique de la pensée ricœurienne n'a pas encore fait l'objet de questionnements au sein d'une théorie de la lecture littéraire en didactique. Notre intention est d'esquisser de tels prolongements dans la seconde partie. Celle-ci comporte trois moments. Dans le premier, nous exposons les termes d'une tension productive entre les notions de sujet lecteur et de sujet éthique. Nous suivons pas à pas le cheminement proposé par Ricœur, dans l'étude VII de Soi-même comme un autre, depuis la constitution de soi (comme lecteur) vers la visée éthique, qu'il définit comme "la visée de la vie bonne, avec et pour les autres, dans des institutions justes ${ }^{6}$ ». Le second moment part du constat de l'oubli de la lecture dans les études VII et VIII du même ouvrage qui sont entièrement consacrées à l'éthique. Nous tentons de combler ce manque à la lumière du dialogue entre Ricœur et les théoriciens du tournant éthique que sont Wayne Booth et Martha Nussbaum, dialogue exposé dans Temps et Récit III. Au terme de ce détour, la conception ricœurienne de l'efficience éthique de la littérature sera clarifiée. Le troisième moment est plus exploratoire. Il consiste à revenir à la théorie didactique de la littérature. Pour ce faire, nous nous éloignons du commentaire de Soi-même comme un autre et nous refaisons le chemin de la visée éthique en l'adossant cette fois à une théorie de la lecture ${ }^{7}$.

\section{Éducation au pluralisme et didactique de la littérature}

Nos contextes scolaires et sociaux sont marqués par la pluralité des valeurs et la diversité des propositions de justesse éthique. Selon le philosophe de l'éducation québécois, Georges Leroux, "la diversité est constitutive de la situation morale et politique contemporaine et de ce fait, elle se situe au cœur des débats sur les finalités de l'éducation ${ }^{8} »$. Leroux distingue, d'une part, la pluralité du pluralisme, et d'autre part, le pluralisme moral du relativisme moral. 


\section{Éduquer au pluralisme : diversité, pluralisme et relativisme moral}

\section{Pluralité et pluralisme}

6 La pluralité ou la diversité sont des faits sociaux indéniables, alors que le pluralisme est une théorie éthique et politique. Selon Leroux, si le pluralisme peut être rejeté au nom d'une autorité, d'une croyance ou d'une doctrine particulière, la pluralité ne peut être niée comme fait. Dans Différence et liberté, il défend l'idée d'une éducation au pluralisme. Le pluralisme recouvre un ensemble de théories distinctes. Le pluralisme est donc luimême un concept pluriel qu'il s'emploie à définir. Depuis la critique de Nietzsche, reprise dans l'herméneutique contemporaine, le débat s'est structuré entre « d'un côté, les tenants d'une vérité universelle, soit en morale soit en religion, dont la position peut être qualifiée de réaliste, la vérité étant considérée comme réelle ; de l'autre, les tenants de positions contraires, les pluralistes et les relativistes, pour qui la pluralité caractérise l'ensemble des doctrines morales et des doctrines épistémologiques qui leur sont associées ${ }^{9} "$.

\section{Pluralisme moral et relativisme}

7 Selon Leroux, le pluralisme moral et le relativisme moral sont des théories philosophiques qui doivent être distinguées :

[Le relativisme moral philosophique] soutient qu'en raison de cette diversité [factuelle] et des facteurs historiques contingents qui déterminent la connaissance morale, aucune morale rationnelle ne saurait être proposée. En conséquence, le relativisme moral affirme que la pluralité est irréductible et qu'il n'existe aucune morale transcendante, universelle ou naturelle, qui soit hors de l'histoire ${ }^{10}$.

Il s'agit donc d'une conception forte de l'historicité de la connaissance, non substantialiste ${ }^{11}$.

9 Le pluralisme moral, comme approche théorique ou normative, repose sur une épistémologie qui tient compte de la diversité des conceptions de la vie bonne au sein de la société et du conflit moral qui en résulte. À la différence du relativisme, le pluralisme ne rejette pas la possibilité d'atteindre le vrai en morale, par le biais de la rationalité.

Le pluralisme moral est nettement distinct du relativisme, en ce qu'il ne doute pas de la possibilité de progresser rationnellement, par le moyen de l'éthique, vers une morale rationnelle, mais il invite à constater qu'historiquement celle-ci n'est jamais achevée ${ }^{12}$.

10 Il s'agit donc d'un substantialisme dit «faible ». En éducation, le pluralisme est conçu comme une norme et comme un idéal à respecter. Il s'agit d'un " pluralisme normatif ». C'est, par exemple, dans cette approche que se situe, selon lui, le concept de laïcité scolaire $^{13}$.

11 Le point commun entre les deux approches réside dans l'impossibilité de s'appuyer sur une norme morale instituée a priori, puisque dans la perspective relativiste cette morale universelle a priori est illusoire, et que, dans la perspective pluraliste, cette vérité morale est un idéal rationnel à poursuivre, un horizon. 


\section{Pluralisme moral et lecture littéraire}

Qu'en est-il des rapports entre l'éducation au pluralisme moral et l'enseignement de la lecture littéraire?

Le pluralisme moral nous semble un enjeu important de l'enseignement de la lecture littéraire, car la pluralité des valeurs se manifeste au moins à trois niveaux : celui du texte, celui de l'activité du lecteur et celui de leur articulation dans un contexte didactique. Au niveau du texte, ou dans une perspective poétique, la littérature problématise la pluralité des valeurs, des normes, des formes de la vie bonne, des manières d'être au monde, de penser, de croire, de sentir. Cette pluralité est refigurée, narrativisée, dialogisée dans l'œuvre littéraire. Au niveau de l'activité du lecteur, ou dans une perspective de la réception, la pluralité des valeurs est à la fois une ressource et un produit de l'acte de lecture. La pluralité morale s'observe, entre autres, sous la forme de "ressources axiologiques" mobilisées par le lecteur, telles que la verbalisation d'une croyance ou d'une position axiologique, le jugement moral sur les personnages, la recherche imaginaire de comportements exemplaires ${ }^{14}$. De plus, le lecteur choisit, parmi les modèles de justesse éthique que lui propose la littérature, celle(s) qui correspond(ent) à " ses possibles les plus propres ${ }^{15}$ ». En ce sens, il peut y avoir un renforcement des valeurs constitutives de l'attestation de soi ou bien une distanciation par rapport à ces valeurs et l'invention d'une autre forme d'attestation de soi.

Dans une approche didactique, il conviendrait d'articuler la pluralité des valeurs représentées dans l'œuvre et la pluralité des valeurs refigurées par les lecteurs, en tenant compte des contextes scolaires dans lesquels le pluralisme moral est institutionnalisé. Ce «pluralisme normatif » (Leroux, 2016) s'observe, par exemple, dans les prescriptions institutionnelles ${ }^{16}$.

15 Ainsi, l'enseignement de la littérature dans une visée d'éducation au pluralisme consisterait à étudier les valeurs dans l'œuvre, à faire exprimer et mettre à distance leurs valeurs par les élèves et à transmettre certaines valeurs par la lecture de l'œuvre. Le problème, à l'évidence, réside dans les probables divergences entre ces trois ensembles de valeurs : le représenté, le refiguré, le prescrit. Considérons l'exemple du choix du corpus. Selon la perspective adoptée, les critères de sélection des œuvres varient. Dans une perspective orientée par la poétique des valeurs, l'enseignant pourrait choisir des œuvres qui présentent une complexité, voire des contradictions entre les systèmes de valeurs attribuables à diverses instances narratives. Dans une perspective centrée sur la réception esthétique, il sélectionnerait des œuvres susceptibles de susciter, par exemple, des réactions axiologiques et de favoriser leur mise à distance par les sujets lecteurs. Dans une perspective normative, il privilégierait des œuvres qui traitent de certaines problématiques ou thématiques et qui transmettent une conception de la « vie bonne » déterminée, dans une certaine mesure par des finalités politiques (par exemple, la justice sociale). L'approche des programmes français actuels semble s'inscrire dans la troisième voie. La limite de cette approche, outre qu'elle fait moins de place à l'étude de la poétique, réside dans la prescription de valeurs déterminées a priori (substantialisme fort) de préférence à la mise en discussion de morales particulières dont les contradictions restent encore largement à résoudre. 


\section{Questions}

La question que nous aborderons dans cet article est la suivante : dans l'enseignement de la littérature, comment faire place au pluralisme moral dans une visée éducative de nature éthique, sans recourir à une substantialisation des valeurs? Cette question à connotation philosophique peut être précisée ainsi : peut-on éduquer au pluralisme moral (représenté dans l'œuvre et refiguré par le lecteur) sans prescrire des valeurs déterminées a priori en fonction d'une morale particulière? Si oui, comment enseigner la lecture littéraire, dans une visée d'éducation au pluralisme moral, qui soit dégagée de tout moralisme a priori?

Notre premier pas dans l'élucidation de cette question a consisté à relire Soi-même comme un autre de Ricœur puisque c'est dans cet ouvrage qu'il a consacré à sa théorie de la lecture une place charnière entre la constitution de la subjectivité (identité narrative) et la visée éthique. Notre but est de prolonger la visée éthique, telle que définie par Ricœur, dans une théorie de la lecture littéraire. Le mouvement de notre réflexion se développera en trois parties :

- De la théorie de la lecture à la visée éthique

- Le dialogue entre Ricœur et les théoriciens du tournant éthique (Booth et Nussbaum)

- Le retour vers la théorie de la lecture littéraire

\section{De la théorie de la lecture à la visée éthique}

Vincent Jouve met en garde contre une instrumentalisation moraliste de la littérature qui ignorerait sa dimension de «fictionnalisation » il prend parti pour l'idée de Ricœur selon laquelle la littérature est un laboratoire d'expériences possibles. Toutefois, pouvons-nous conclure avec lui que « cette expérience de réalité fictive ne nous engage pas en tant que sujet du monde ${ }^{17} »$ ? Du point de vue de l'expérience subjective de lecture, la ligne de partage entre le sujet lecteur et le "sujet du monde » est-elle théoriquement pertinente ? La posture du lecteur critique déjouant les chausse-trappes axiologiques dans le texte littéraire peut-elle être entièrement coupée de la constitution de soi comme sujet de l'imputation morale?

\section{Du sujet lecteur au sujet éthique}

19 Sur le plan de la constitution de soi, il importe de distinguer, mais non d'opposer, la part du sujet lecteur de celle du sujet éthique. À cette fin, une relecture de Ricœur est particulièrement éclairante, car il montre que l'éthique et la théorie de la lecture entretiennent des relations de complémentarité qui ne sont pas exemptes de tensions.

\section{Le sujet lecteur : le versant imaginatif et critique du sujet éthique ?}

Selon Ricœur, aucun récit n'est éthiquement neutre, et il appartient au lecteur, en dernière instance, "de choisir entre les multiples propositions de justesse éthique véhiculée par la lecture ${ }^{18} »$. En choisissant, entre autres, parmi ces propositions, le lecteur constitue en partie son identité narrative. La constitution de l'identité narrative est dynamique car elle procède d'une tension entre ce qui se maintient (mêmeté) et ce qui se transforme (ipséité). Selon Ricœur, la pluralité et la mobilité (ipséité) que permet 
la lecture "corrige", conteste, atténue la prétention de l'éthique normative à régir seule la subjectivité. Autrement dit, l'imagination narrative vient perturber, modaliser, reporter dans un horizon plus lointain, la constitution de soi comme sujet de l'imputation morale et de l'action. Le sujet lecteur serait en quelque sorte le versant imaginatif et critique du sujet éthique. Grâce à la lecture, le sujet est emporté vers un devenir autre. Or, ce devenir peut consister à reconsidérer les implications de sa morale particulière et de son action dans la communauté. C'est donc bien en tant que sujet éthique que le lecteur s'engage dans le processus de la lecture.

\section{Le sujet éthique : garde-fou du sujet lecteur?}

21 D'autre part, dans Soi-même comme un autre, le risque de dissolution de l'identité narrative est dépassé grâce à l'introduction de la notion éthique du maintien de soi, liée à la promesse faite à autrui ${ }^{19}$. À son tour, l'ouverture à la possibilité de devenir autre que permet la lecture est limitée, corrigée, encadrée par la responsabilité éthique du maintien de soi. Le maintien de soi dans le temps de la lecture, comme dans celui de la promesse, est la catégorie que Ricœur introduit pour éviter la dissolution du sujet dans la multiplicité des variations imaginatives. Finalement, la mêmeté (ce qui dans le soi se maintient) est donc à considérer comme une catégorie éthique, liée à la responsabilité, plutôt que comme une caractéristique intrinsèque du sujet (lecteur).

Ainsi, l'imagination narrative, à l'œuvre dans la lecture, constitue le versant imaginatif, créatif, critique du sujet éthique ; alors que la responsabilité éthique permet au sujet lecteur de conserver ce qui en lui se maintient, en dépit des transformations imprimées par ses lectures.

\section{La visée éthique}

La constitution de soi comme lecteur n'épuise évidemment pas la question des rapports entre la littérature et la visée éthique, définie par Ricœur comme «la visée de la vie bonne, avec et pour les autres, dans des institutions justes ${ }^{20} »$. Comment le philosophe articule-t-il la constitution de soi à la visée de la vie bonne ? C'est l'objet de la septième étude de Soi-même comme un autre intitulée «Le soi et la visée éthique». Le cheminement emprunté par Ricœur comporte trois mouvements, qui correspondent à trois dimensions (individuelle, interpersonnelle et sociétale) de la visée éthique : la constitution de soi, la sollicitude envers autrui (dans le dialogue interpersonnel entre un « je » et un « tu») et la reconnaissance de la pluralité des tiers au sein d'institutions justes $^{21}$.

\section{La constitution de soi}

24 La constitution de soi repose sur la catégorie morale de l'estime de soi (attestation, imputabilité, promesse). Ce qui nous intéresse en tant que didacticienne de la littérature, c'est le parallèle explicite que Ricœur établit entre une catégorie de la théorie de la lecture - l'interprétation de soi - et la catégorie correspondante dans sa théorie éthique - l'estime de soi. En effet, pour Ricœur, la vie bonne est un travail de nature interprétative. Il s'agit en quelque sorte de lire le texte de sa propre vie. Interpréter le texte de nos actions, c'est nous interpréter nous-mêmes. Ainsi, « au plan éthique, l'interprétation de soi devient estime de $\operatorname{soi}^{22} »$. Tout comme l'interprétation 
littéraire, l'interprétation de soi peut au mieux se prévaloir, du moins aux yeux des autres, de la plausibilité, et cela, même si aux yeux du sujet, sa propre conviction confine à une sorte d'évidence expérientielle.

\section{La sollicitude envers autrui}

La sollicitude envers autrui repose sur la catégorie de la réciprocité. Il s'agit dans ce deuxième mouvement de considérer autrui comme un autre soi-même. Est convoqué le modèle de la philia aristotélicienne, de l'amitié, comme réciprocité entre un «je » et un « tu ». La réflexion se situe ici sur le plan interpersonnel, intime. Il est à noter que le "vivre-ensemble» est d'abord situé par Ricœur sur ce plan interpersonnel de la réciprocité.

\section{La reconnaissance de la pluralité des tiers}

Le vivre ensemble, le vivre bien, ne se limite pas aux relations interpersonnelles, il s'étend à la vie commune garantie par des institutions. Par "institution ", Ricœur entend la "structure du vivre-ensemble d'une communauté historique ${ }^{23}$ ». Le troisième mouvement est celui de la justice qui marque le passage de la sollicitude envers un « tu » (le sujet qui a un visage) à la reconnaissance du « tiers » (le sujet construit par la loi). Cette reconnaissance ne peut être garantie que par des institutions justes. La justice présente une exigence nouvelle, qui n'est pas contenue dans la sollicitude, une exigence d'égalité. L'enjeu est éminemment politique et il requiert au moins deux conditions : la reconnaissance de la pluralité et la concertation. Selon Ricœur, «l'idée de pluralité suggère l'extension des rapports humains à tous ceux que le face-à-face entre un "je" et un "tu" laisse en dehors au titre de tiers ${ }^{24} »$. Tout l'enjeu est de reconnaître "la pluralité des tiers qui ne seront jamais des visages ${ }^{25}$ ». Quant à la concertation, elle est d'ordinaire invisible, car elle est recouverte par les relations de dominations. Dans l'histoire, elle apparait toujours de façon disruptive et discontinue lorsqu'elle est sur le point d'être ruinée et de laisser le champ libre à la violence.

Pour la recherche en didactique de la littérature, un enjeu majeur de la problématisation de la visée éthique pourrait être de comprendre l'oscillation entre la constitution de soi-même comme lecteur et la reconnaissance des tiers. Or, la description de la visée éthique dans Soi-même comme un autre s'accompagne d'un abandon de la théorie de la lecture (dans les études VII et VIII). L'entrée dans la réflexion philosophique proprement éthique s'accompagnerait-elle d'une sortie hors du champ de la littérature?

\section{Le dialogue entre Ricœur et les théoriciens du tournant éthique}

Penser le sujet éthique, serait-ce oublier le sujet lecteur ${ }^{26}$ ? Pour interpréter ce manque, une piste consiste à revenir à la théorie de la lecture exposée par Ricœur dans Temps et récit III $^{27}$. Dans cet ouvrage, Ricœur entame un dialogue très instructif avec les plus éminents précurseurs du «tournant éthique » en littérature que sont Wayne Booth et Martha Nussbaum. 
29 Le «tournant éthique " dans la théorie littéraire doit beaucoup aux travaux anglophones de Martha Nussbaum et de Wayne Booth ${ }^{28}$. Le tournant éthique a permis de dépasser l'opposition entre esthétique et éthique et de sortir d'un formalisme oublieux du lecteur. En cela, les rapprochements avec la théorie didactique du sujet lecteur semblent fructueux. Toutefois, l'utilisation de la littérature aux fins du discours de l'éthique a été discutée. Dans le champ de la critique littéraire, Korthals Altes met en garde contre la possible réduction, par la critique éthique, de la lecture littéraire à la résolution de dilemmes moraux ${ }^{29}$. Cette mise en garde est-elle justifiée? Prenons deux exemples de réponses adressées par Ricœur, l'une à Booth, l'autre à Nussbaum.

\section{Les canailles séduisantes et le lecteur mystifié}

Booth propose de sélectionner et de hiérarchiser les textes en fonction d'une norme morale $^{30}$; autrement dit, un glissement s'opère de la visée éthique de la lecture à la caractérisation du corpus selon des valeurs a priori. Toutefois, Booth a le mérite de poser la question délicate des œuvres qui sont à la fois transgressives sur le plan moral et mystificatrices à l'égard du lecteur. Le modèle de la lecture de Booth est fondé sur l'amitié entre le texte et le lecteur (la philia aristotélicienne), ce qui le conduit à rejeter les récits qui présentent des points de vue indiscernables et des narrateurs indignes de confiance, ceux que Ricœur nomme joliment « les canailles séduisantes ». Selon Booth, ces derniers sont capables d'entraîner le lecteur dans la plus grande confusion morale. Il condamne par exemple La Chute d'Albert Camus, susceptible, selon lui, d'entraîner le lecteur dans l'effondrement spirituel de Clamence. Ricœur commente ainsi cette position de Booth :

Il peut être justifié à craindre qu'un lecteur plongé dans la confusion, mystifié, bafoué [...] soit insidieusement invité à renoncer à la tâche assignée à la narration par Érich Auerbach : celle de conférer ordre et signification à nos vies. Le danger est en effet que la persuasion cède la place à la séduction de la perversité. C'est le problème posé par les « canailles séduisantes » que sont les narrateurs d'une bonne partie de la littérature contemporaine. Par-dessus tout, Booth a raison de souligner, à l'encontre de toute esthétique prétendue neutre, que la vision des personnages communiquée et imposée au lecteur, a des aspects non seulement psychologiques et esthétiques, mais sociaux et moraux. [...] On peut toutefois se demander qui est juge de ce qui est ultimement pernicieux ${ }^{31}$.

Dans cet extrait se dégage une thèse que Ricœur réaffirme en maints passages de Temps et récit III, à savoir que la théorie de la lecture doit être absolument dégagée de tout moralisme.

\section{Un lecteur qui répond et qui (se) questionne}

\section{Un lecteur qui répond}

Ricœur concède que la littérature moderne puisse être dangereuse, mais selon lui "cette littérature vénéneuse requiert un nouveau type de lecteur: un lecteur qui répond ${ }^{32}$ ». Moins le narrateur s'avère digne de confiance, plus l'entreprise de séduction se dissimule, plus la littérature se fait corrosive, plus la liberté et la responsabilité du lecteur sont requises. Autrement dit, l'exemple des canailles séduisantes illustre la nécessité de former des lecteurs distancés et réflexifs. Dans un ouvrage antérieur, $D u$ 
texte à l'action. Essai d'herméneutique II, Ricœur explicitait déjà le rapport entre une narration perverse et la distanciation du lecteur.

Même la plus pernicieuse, la plus perverse entreprise de séduction (celle par exemple qui rendra estimable l'avilissement de la femme, la cruauté et la torture, la discrimination raciale [...]) peut, à la limite, revêtir au plan de l'imaginaire une fonction éthique : celle de la distanciation ${ }^{33}$.

La visée éthique n'est donc pas à chercher dans des valeurs universellement estimables, qui seraient proposées (ou imposées) au lecteur, mais dans la complexité des instances narratives, dans le caractère dialogisé, narrativisé, du pluralisme moral qui renvoie le lecteur à sa propre liberté, à sa propre responsabilité, à la mise à distance de son imagination éthique.

\section{Un lecteur qui questionne}

Kortals Altes entrevoit dans les propositions de Nussbaum un risque d'inféoder la lecture à un «but moral pratique », puisque selon sa lecture de Love's Knowledge (1990) la littérature devrait contribuer à répondre à la question comment vivre? Pour Kortals Altes, la recherche d'une réponse cohérente et partageable à cette question courrait le risque de soumettre la lecture à l'édification morale. La problématique ici soulevée est cruciale, car elle concerne l'efficience attribuée à la littérature en regard de la formation du lecteur dans une visée éthique. Ricœur propose une hypothèse, héritée de Gadamer :

Le moment où la littérature atteint son efficience la plus haute est peut-être celui où elle met le lecteur dans la situation de recevoir une solution pour laquelle il doit lui-même trouver les questions appropriées, celles qui constituent le problème esthétique et moral posé par l'œuvre ${ }^{34}$.

Pour Ricœur, la littérature n'offre pas de réponses, elle offre des questions, or tout surgissement d'interrogations met en question le déjà-là. Ce surgissement d'interrogations constitue paradoxalement l'efficience de la littérature : « Le défaut de réponse aux dilemmes d'une époque est peut-être l'arme la plus efficace dont dispose la littérature pour agir sur les mœurs et changer la pratique ${ }^{35}$ ».

Si les textes littéraires ne délivrent pas une connaissance pratique susceptible d'orienter la délibération morale, ce n'est pas parce qu'ils sont en défaut par rapport à une sagesse pratique, mais parce qu'ils excèdent les termes même dans lesquels cette sagesse pratique est énoncée dans une communauté et à une époque donnée. L'imagination éthique peut être interprétée comme le pendant éthique de l'imagination narrative dans une théorie de la lecture ${ }^{36}$.

\section{Le retour à la théorie de la lecture littéraire : vers une éthique de la lecture?}

Le troisième mouvement de notre réflexion, celui du retour de la visée éthique vers la théorie de la lecture, est le plus exploratoire. Dans Soi-même comme un autre, après les deux études consacrées à l'éthique et à la norme morale, qui, nous le rappelons, sont marquées par l'absence de la lecture et du lecteur, Ricœur opère un retour qualifié d'« intempestif » à la littérature. Dans un surprenant "interlude », il livre sa propre lecture d'Antigone de Sophocle signalant ainsi ce qui, dans la tragédie, «instruit 
l'éthique ${ }^{37}$ ». Ce retour à la théorie de la lecture est à peine esquissé par Ricœur. Il peut, au mieux, être inféré à partir de sa lecture d'Antigone ${ }^{38}$. Pour tenter de le prolonger, refaisons le chemin de la visée éthique, qui nous a amené de la constitution de soimême à la reconnaissance de la pluralité des tiers, en l'adossant cette fois explicitement à une théorie de la lecture littéraire.

\section{La constitution de soi}

Le premier jalon de la réflexion sur la visée éthique est la constitution de soi, qui est un travail de nature interprétative. Comme nous l'avons vu, Ricœur identifie de manière explicite interprétation de soi et estime de soi $^{39}$. Le prolongement dans une éthique de la lecture littéraire est donc assez aisé. Apprendre à interpréter des textes littéraires serait une activité de même nature qu'apprendre à interpréter le texte de ses propres actions. Toutefois, lire n'est pas vivre, ou plutôt lire est un mode particulier du vivre, il s'agit de vivre sur le mode du possible. «La fiction soumet notre jugement moral à des variations imaginatives ${ }^{40} »$. La refiguration des valeurs du lecteur repose ainsi sur la suspension temporaire du jugement moral et l'ouverture à d'autres manières d'être possibles. Le premier prolongement éthique de la lecture est inhérent au fonctionnement de l'imagination narrative. La lecture participe à la constitution de soi comme sujet du monde, en cela qu'elle mobilise l'imagination éthique. De plus, l'interprétation de soi, et son pendant éthique l'estime de soi, ne peut au mieux se prévaloir que de la plausibilité et non d'une quelconque vérité. On mesurera ainsi, par exemple, l'importance de penser, dans l'enseignement, la discussion sur la plausibilité d'une interprétation littéraire pour la formation éthique du sujet lecteur.

\section{La sollicitude envers autrui}

Le second jalon est celui de la sollicitude d'un « je » en regard d'un « tu » qui repose sur la catégorie de la réciprocité. Il s'agit de considérer autrui comme un autre soi-même. Le prolongement dans une théorie de la lecture est ici plus exploratoire. Comment s'effectue la sollicitude envers autrui dans la lecture et de quel «autrui » s'agit-il ? S'agit-il d'interpréter le texte des actions et des discours d'un personnage dans une œuvre? Ou s'agit-il de reconnaître autrui comme un autre sujet lecteur, comme un interprète amical ? Ricœur ne le dit pas. Les deux versants de la sollicitude, de la philia, sont certainement complémentaires, toutefois il convient de les distinguer comme l'a montré l'exemple des « canailles séduisantes ».

Dans une visée éthique de l'enseignement de la littérature, il serait intéressant d'étudier la réciprocité entre constitution de soi (soi-même comme un autre, soi-même comme un sujet lecteur autre) et reconnaissance d'autrui (comme un autre soi-même, comme un autre sujet lecteur). Ce serait le deuxième prolongement éthique de la lecture. Le dialogue régulé entre sujets lecteurs permet sans doute de favoriser cette réciprocité, mais n'est certainement pas suffisant. 


\section{La reconnaissance de la pluralité des tiers dans des institutions justes}

41 pertinent sur le plan social. En quoi la lecture permet-elle de reconnaître la pluralité des tiers? A priori rien ne semble garantir le passage systématique de la réciprocité entre les lecteurs dans la classe à « la reconnaissance des tiers qui ne seront jamais des visages $^{41}$ ». Constater la limite de toute tentative pour reconstruire le lien social sur la base d'une relation strictement dialogale, fût-elle empreinte de sollicitude ou d'empathie, est essentiel pour dépasser les facilités d'un discours convenu sur le vivre ensemble. Ce passage, s'il a lieu, ne peut se réaliser que de manière indirecte, provisoire, précaire. Comment le penser? On peut d'abord mettre à l'épreuve de la réflexion didactique les deux catégories à la base de cette reconnaissance : la pluralité et la concertation. Sur le plan de la lecture, la pluralité et son corollaire, la mutabilité, s'observent au travers de la pluralité des interprétations - ce que nous appelons la diversité interprétative. Nous avons observé que la diversité interprétative peut à certaines conditions permettre aux lecteurs d'élargir leur compréhension de la diversité subjective, mais que cette compréhension est incertaine, fictionnelle, relative, car elle très fortement contextualisée dans la situation didactique dans laquelle elle se donne à entendre ${ }^{42}$.

Quant à la concertation, rappelons que, selon Ricœur, elle est d'ordinaire invisible car elle est recouverte par les relations de dominations, et qu'elle n'apparait que de façon discontinue lorsqu'elle est sur le point d'être ruinée. Sur le plan de la lecture, c'est donc dans le conflit des interprétations, que la concertation peut apparaitre, souvent de façon disruptive, imprévisible. En classe, la plupart du temps la concertation est recouverte par des relations de savoir-pouvoir (pour paraphraser Foucault) qui structurent les échanges entre les lecteurs. La relation pédagogique entre l'enseignant et les élèves est une forme particulièrement institutionnalisée de domination discursive dans laquelle la prétention au savoir lire (l'interprétation magistrale ou canonique) légitime le pouvoir dire. De façon plus générale, les communautés interprétatives, en cela qu'elles dépassent la relation dialogale dans la classe et font circuler des normes et des valeurs relatives au fonctionnement interprétatif pourraient être pertinentes pour étudier ce passage de la réciprocité interpersonnelle à la reconnaissance intersubjective. Faudrait-il encore que ces communautés soient pensées selon le principe de l'égalité, qui dans le cas de la lecture est moins une catégorie juridique, qu'un principe éducatif, à savoir la reconnaissance de l'égalité des compétences interprétatives ${ }^{43}$.

\section{Conclusion}

43 Le point de départ de cette réflexion est une préoccupation pour penser l'enseignement de la littérature dans une visée d'éducation au pluralisme moral, sur des fondements épistémologiques dégagés de tout moralisme a priori. Le pluralisme normatif qui prévaut dans certaines prescriptions institutionnelles pour l'éducation (MELS, MEN, UNESCO) constitue une avancée en ce qui concerne la prise en compte de la diversité des conceptions éthiques et des morales particulières auxquelles les élèves peuvent adhérer ${ }^{44}$. Cependant, il semble limité, en cela qu'il privilégie la transmission de valeurs 
a priori, dans le but de constituer une morale universellement acceptée. De plus, cette approche ne problématise pas suffisamment la spécificité de l'enseignement de la littérature pour la formation éthique des sujets lecteurs.

L'herméneutique de Ricœur nous semble pouvoir constituer un fondement théorique pour mener à bien une telle entreprise, car elle accorde une place charnière à la théorie de la lecture entre la constitution du sujet (lecteur) et la visée éthique. Toutefois, après les études consacrées à l'éthique dans Soi-même comme un autre, Ricœur n'effectue pas un retour explicite à la théorie de la lecture, même si celui-ci est esquissé dans l'interlude consacré à la tragédie grecque. Nous avons tenté de prolonger cette esquisse en nous référant au dialogue entre Ricœur et deux théoriciens du tournant éthique dans Temps et Récit III. Ce détour nous a permis d'interpréter l'irruption de la lecture singulière d'Antigone ${ }^{45}$, comme une réponse de Ricœur à une conception déficitaire de la littérature par rapport à une sagesse pratique. Si les textes littéraires ne délivrent pas de réponses pratiques susceptibles d'orienter la délibération morale, ce n'est pas parce qu'ils sont en défaut par rapport à une sagesse pratique, mais parce qu'ils excèdent les termes mêmes dans lesquels cette sagesse pratique s'énonce. Cette conception de la littérature permet d'entrevoir son enseignement dans une visée éthique, qui soit dégagé de tout moralisme a priori.

Si l'enseignement de la littérature peut contribuer à une visée éthique de l'éducation au pluralisme, ce ne devrait pas être au prix de ce qui la constitue en propre, à savoir le déploiement d'un mouvement compréhensif et conflictuel entre soi, autrui et la pluralité des tiers, mouvement qui relève de formes du possible, de manières d'êtres fictionnelles, voire encore à inventer. Parce qu'elle requiert des lecteurs qui questionnent, non seulement les valeurs véhiculées par les textes, mais aussi les valeurs qui constituent le tissu des évidences partagées au sein de leurs communautés, la lecture littéraire appelle plus que la constitution de soi grâce à l'imagination narrative. Elle appelle l'invention d'autres rapports à la pluralité des lecteurs qui ne seront jamais des visages. Elle appelle une éducation de l'imagination éthique.

\section{NOTES}

1. P. Ricœur, Temps et Récit III, Le temps raconté, Paris, Seuil, coll. « Points », 1985, p. 317.

2. Nous empruntons cette distinction entre enseigner l'éthique par la littérature, enseigner l'éthique de la littérature ou enseigner une lecture éthique à Claude Puidoyeux, «Le tournant éthique de l'enseignement de la littérature, quelques questions ", Repères $n^{\circ} 58$, p. 15-30, déc. 2018, et nous reformulons librement. [En ligne]: <https://journals.openedition.org/reperes/ 1664>

3. Cette réflexion s'inscrit dans la continuité de nos travaux sur les fondements herméneutiques d'un enseignement de la lecture qui prenne acte de la diversité culturelle, épistémique et morale des sujets lecteurs en contexte scolaire. Elle est donc teintée par nos propres préoccupations et croyances.

4. G. Leroux, Différence et liberté, Enjeux actuels de l'éducation au pluralisme, Montréal, Boréal, 2016.

5. P. Ricœur, Soi-même comme un autre, Paris, Seuil, coll. « Points », 1990. 
6. Ibid., p. 202.

7. Ce choix de présentation pourrait laisser entendre que nous proposons une démarche étapiste. Il n'en est rien, le cheminement proposé est celui d'une réflexion qui s'élabore en lien étroit avec la relecture de Soi-même comme un autre, non celui d'une proposition praxéologique.

8. G. Leroux, Différence et liberté, ouvr. cité, p. 133.

9. Ibid., p. 136.

10. Ibid., p. 156.

11. Leroux distingue également le relativisme épistémique du relativisme moral. Dans la pensée éducative, le relativisme épistémique - à savoir l'idée selon laquelle aucune épistémologie et a fortiori aucune théorie ne peut prétendre à elle seule rendre compte de la complexité du réel semble faire davantage consensus que le relativisme moral - à savoir l'idée qu'aucune morale particulière, fusse-t-elle celle de l'institution scolaire, ne peut prétendre rendre compte de la complexité du social.

12. Id.

13. Ibid., p. 157.

14. Voir M. Sauvaire, É. Falardeau, "Susciter le moment critique. De l'investissement de ressources axiologiques à leur mise à distance par des sujets lecteurs élèves ", Arborescences: Revue d'études françaises, $\mathrm{n}^{\circ}$ 6, p. 121-147.

15. P. Ricœur, Temps et récit III, ouvr. cité, p. 115.

16. Leroux, ouvr. cité.

17. V. Jouve, «Valeurs littéraires et valeurs morales : la critique éthique en question », Les Carnets du Crimel, 2014. [En ligne] : <https://forigin.hypotheses.org/wpcontent/blogs.dir/1449/files/ 2014/03/LitVal_Jouve.pdf>

18. P. Ricœur, Du texte à l'action, Paris, Seuil, 1986, p. 447.

19. Ouvr. cité, p. 149.

20. Ibid., p. 202.

21. Nous présentons les notions dans l'ordre où elles apparaissent dans le texte de Ricœur. Une interprétation linéaire de la visée éthique serait indue, car les trois dimensions sont intrinsèquement reliées. Par exemple, l'estime de soi comme fondement de la sollicitude vient de la dimension dialogale de la constitution de soi.

22. Ibid., p. 211.

23. Ibid., p. 227.

24. Ibid., p. 228.

25. Id.

26. La question est loin d'être rhétorique, si l'on songe à l'usage prescrit de la littérature dans d'autres disciplines scolaires. En particulier celles qui reposent traditionnellement sur l'éthique, comme l'Éthique et culture religieuse (Ministère de l'Éducation, du Loisir et du Sport, MELS, Québec) ou l'Enseignement civique et moral (Ministère de l'Éducation Nationale, MEN, France). Pour s'en tenir à ces deux exemples, les programmes présentent les œuvres littéraires comme des répertoires de cas particuliers ou de situations problématiques au même titre que des situations vécues de la vie sociale ou scolaire. En tant que didacticienne de la littérature, il nous semble important de signaler le risque d'une instrumentalisation de la littérature aux fins d'une éthique normative.

27. P. Ricœur, Temps et Récit III, ouvr. cité.

28. Voir M. Nussbaum, La Connaissance de l'amour - Essais sur la philosophie et la littérature, trad. S. Chavel, Paris, Les Éditions du Cerf, 2010 et W. Booth, The Company we keep. An Ethics of Fiction, Oakland, University of California Press, 1988.

29. L. Korthals Altes, «Le tournant éthique dans la théorie littéraire : impasse ou ouverture? ", Études littéraires, vol. 31, nº 3, 1999, p. 39-56.

30. Ibid., p. 47. 
31. Temps et récit III, ouvr. cité, p. 295.

32. Soi-même comme un autre, ouvr. cité, p. 296.

33. Ouvr. cité, p. 295.

34. Ibid., p. 317.

35. Id., p. 317.

36. Ricœur n'utilise pas l'expression « imagination éthique ». Nous proposons de nommer ainsi le pendant éthique de l'imagination narrative dans la constitution de soi-même comme lecteur. Nous soulignons ainsi, premièrement, que le sujet lecteur qui répond et qui questionne le « monde du texte » s'engage comme « sujet du monde », deuxièmement, que la constitution de soi (comme sujet lecteur et comme sujet éthique) est de nature imaginative. Tout comme l'imagination narrative, l'imagination éthique est médiatisée par les œuvres; à ce titre elle est fragmentaire, fictionnelle, mouvante et potentiellement contradictoire.

37. Ricœur, Soi-même comme un autre, ouvr. cité, p. 281.

38. Il est notable que la principale théoricienne à laquelle Ricœur se réfère pour étayer son interprétation de Sophocle soit Martha Nussbaum.

39. Ibid., p. 211.

40. Ibid., p. 167.

41. Ibid., p. 228.

42. Voir M. Sauvaire, Diversité des lectures littéraires. Comment former des sujets lecteurs divers?, Thèse de doctorat, Universités de Toulouse-Le Mirail et Laval, 2013. [En ligne]: <http:// www.theses.ulaval.ca/2013/29976/>

43. Voir la démonstration de la pertinence de cette idée dans l'ouvrage d'Yves Citton, Pour une interprétation littéraire des controverses scientifiques, Versailles, éditions Quae, 2013.

44. Voir G. Leroux, ouvr. cité.

45. P. Ricœur, Soi-même comme un autre, ouvr. cité.

\section{RÉSUMÉS}

Le rejet d'une conception formaliste de la lecture comme jeu poétique sur les valeurs textuelles qui n'engagerait pas le lecteur comme sujet éthique, la critique d'un retour possible de l'instrumentalisation moralisante de la littérature, les réserves soulevées par une approche relativiste de la diversité interprétative sont autant de préoccupations exprimées à propos de l'enseignement de la littérature dans une visée éthique. Nous les examinons sous un angle épistémologique, à la lumière des rapports entre théories de la lecture et conceptions d'une éducation au pluralisme moral. Sur quels fondements penser un enseignement de la lecture littéraire dégagé de tout moralisme a priori? Une relecture de Soi-même comme un autre, dans lequel Ricœur consacre à sa théorie de la lecture une place charnière entre la constitution de soi et la visée éthique, nous invite à interpréter l'imagination éthique comme le pendant de l'imagination narrative dans une théorie de la lecture.

Some concerns expressed about teaching literature with an ethical objective are the rejection of a formalist conception of reading as a poetic play of textual value that would not engage the reader as an ethical subject, the criticism of a possible return of literature as a moralization tool, the reservations raised by a relativistic approach of interpretive diversity. From an epistemological angle, we examine these issues focusing on the relations between the theories of 
reading and the conceptions of moral pluralism in education. Which theoretical foundation could we use for teaching literary reading on an ethical purpose absolve of all moralism a priori? A rereading of "Self As Another," in which Ricœur devotes the theory of reading a pivotal place between self-constitution and the ethical will, invites us to interpret the ethical imagination as the instant of the narrative imagination in reading theory.

\section{AUTEUR}

\section{MARION SAUVAIRE}

Marion Sauvaire est professeure de didactique du français à l'université Laval de Québec, Canada. Ses recherches portent sur la didactique de la littérature dans l'enseignement secondaire et supérieur et sur la théorie de la lecture littéraire. Elle a codirigé le numéro 58 de la revue Repères intitulé « Le tournant éthique en didactique de la littérature » (2018). Elle a publié également avec Érick Falardeau « Susciter le moment critique. De l'investissement de ressources axiologiques à leur mise à distance par des sujets lecteurs élèves » (in Arborescence $\mathrm{n}^{\circ}$ 6, Voix et valeurs du discours littéraire, 2016). 